30(5), 647-663

\title{
A generalized likelihood ratio chart for monitoring type I right-censored Weibull lifetimes
}

\author{
Sung Won $\operatorname{Han}^{a} \cdot$ Jaeheon Lee ${ }^{a, 1}$ \\ ${ }^{a}$ Department of Applied Statistics, Chung-Ang University \\ (Received July 17, 2017; Revised August 23, 2017; Accepted August 23, 2017)
}

\begin{abstract}
Weibull distribution is a popular distribution for modeling lifetimes because it reflects the characteristics of failure adequately and it models either increasing or decreasing failure rates simply. It is a standard method of the lifetimes test to wait until all samples failed; however, censoring can occur due to some realistic limitations. In this paper, we propose a generalized likelihood ratio (GLR) chart to monitor changes in the scale parameter for type I right-censored Weibull lifetime data. We also compare the performance of the proposed GLR chart with two CUSUM charts proposed earlier using average run length (ARL). Simulation results show that the Weibull GLR chart is effective to detect a wide range of shift sizes when the shape parameter and sample size are large and the censoring rate is not too high.
\end{abstract}

Keywords: ARL, generalized likelihood ratio chart, type I right-censored data, Weibull distribution

\section{1. 서론}

통계적 공정관리(statistical process control; SPC)는 통계적인 방법을 사용하여 제조공정을 관리하는 절차를 총칭하고 있으며, 관리도(control chart)는 $\mathrm{SPC}$ 에서 가장 널리 사용되는 도구이다. 관리도를 운 용하기 위해서는 먼저 제품의 품질특성치(quality characteristic)를 측정해야 하는데, 제품의 경우 길 이, 무게, 온도, 강도, 수명 등 다양한 품질특성치들이 존재한다. 이 논문에서는 제품의 품질특성치로서 제품의 수명을 고려하였다.

제품의 수명시험(lifetimes test)은 기본적으로 표본을 구성하는 $n(n \geq 1)$ 개의 제품이 모두 고장날 때 까지 진행하게 된다. 그러나 시간적이나 비용적인 제약 때문에 모든 제품이 고장날 때까지 시험하는 것 이 어려운 경우가 많으며, 이러한 경우 인위적으로 시험을 중도절단해서 수명 데이터를 수집하는 경우 가 빈번하게 발생한다. 이와 같이 시험을 시작한 후 일정 기간이 지나고 중도절단하는 것을 우측중도 절단(right censoring)이라 부르며, 제 1 형 우측중도절단(type I right censoring)과 제 2 형 우측중도절 단(type II right censoring)으로 구분할 수 있다. 제 1 형 우측중도절단은 사전에 정해진 시점까지 시험

This research was supported by the Chung-Ang University Graduate Research Scholarship in 2016, and supported by Basic Science Research Program through the National Research Foundation of Korea (NRF) funded by the Ministry of Education (2017R1D1A1B03029035).

${ }^{1}$ Corresponding author: Department of Applied Statistics, Chung-Ang University, 84 Heukseok-ro, Dongjakgu, Seoul 06974, Korea. E-mail: jaeheon@cau.ac.kr 
을 진행한 후 중단하는 것이고, 제 2 형 우측중도절단은 사전에 정한 개수만큼 제품이 고장 난 시점에서 시험을 중단하는 것이다. 이 논문에서는 산업 현장에서 일반적으로 발생하는 제 1 형 우측중도절단된 수 명 데이터를 고려하였다.

와이블 분포(Weibull distribution)는 지수 분포(exponential distribution)를 일반화한 분포로서 스 웨덴의 물리학자인 Weibull이 처음 제안하였으며 제품의 수명 모형으로 잘 적합된다고 알려져 있다 (Lieblein과 Zelen, 1956; Kao, 1959; Lawless, 2003). 제품의 수명 데이터를 적합시키는 경우 일반적 으로 2 모수 와이블 분포인 $\operatorname{Weibull}(\beta, \eta)$ 를 사용하며, 여기서 $\beta$ 는 형상모수(shape parameter)이고 $\eta$ 는 척도모수(scale parameter)를 나타낸다. 와이블 분포는 형상모수 $\beta$ 에 따라 다양한 형태를 가지는데, 대 부분의 제품의 경우 $\beta$ 는 0.5 에서 5 사이의 값을 갖는다고 알려져 있다 (Olteanu, 2010). 즉, $\beta=1$ 인 경 우 상수의 고장률(failure rate), $\beta<1$ 인 경우 감소하는 고장률, 그리고 $\beta>1$ 인 경우 증가하는 고장률 을 갖는다는 특징이 있다. 척도모수 $\eta$ 는 와이블 분포를 따르는 제품의 약 $63.2 \%$ 가 고장나는 시간을 나 타내며, 따라서 $\eta$ 를 특성수명(characteristic life)이라고 한다.

와이블 분포의 확률밀도함수(probability density function; pdf)와 누적분포함수(cumulative distribution function; cdf)는

$$
\begin{aligned}
& f(t \mid \beta, \eta)=\frac{\beta}{\eta}\left(\frac{t}{\eta}\right)^{\beta-1} \exp \left[-\left(\frac{t}{\eta}\right)^{\beta}\right], \quad t>0, \\
& F(t \mid \beta, \eta)=1-\exp \left[-\left(\frac{t}{\eta}\right)^{\beta}\right], \quad t>0
\end{aligned}
$$

이다. 이때 분포의 평균은 $\eta \Gamma(1+1 / \beta)$ 이고, 미리 정해진 중도절단시점을 $C$ 라 할 경우 중도절단 율(censoring rate) $\mathrm{pc}$ 는

$$
\mathrm{pc}=\operatorname{Pr}(T>C)=\exp \left[-\left(\frac{C}{\eta}\right)^{\beta}\right]
$$

가 됨을 쉽게 알 수 있다.

와이블 분포의 두 가지 모수 $\beta$ 와 $\eta$ 의 변화를 탐지하는 관리도 절차에 대한 연구가 많이 진행되었는데, 형상모수인 $\beta$ 의 변화보다 제품의 평균수명에 좀 더 많은 영향을 미치는 척도모수 $\eta$ 의 변화에 대해 더 많은 연구가 진행되어 왔다. $\beta$ 가 고정되어 있고 $\eta$ 가 변화하는 경우 이를 탐지하기 위해 제 1 형 우측중도 절단된 와이블 데이터를 모니터링하는 절차에 대한 대표적인 연구는 다음과 같다. Steiner와 MacKay (2001)와 Zhang과 Chen (2004)은 조건부 기댓값의 개념을 적용한 exponentially weighted moving average (EWMA) 관리도를 제안하였고, 최근 Dickinson 등 (2014)은 cumulative sum (CUSUM) 관 리도를 제안하고 Zhang과 Chen (2004)이 제안한 EWMA 관리도와 그 효율을 비교하였다. Dickinson 등 (2014)은 모의실험을 통해 그들이 제안한 관리도가 Zhang과 Chen (2004)의 관리도보다 전반 적으로 성능이 더 우수함을 보였다. Choi와 Lee (2016)는 이산형 관리도의 형태인 binomial CUSUM (BCUSUM) 관리도를 제안하고 그 효율을 Dickinson 등 (2014)의 CUSUM 관리도와 비교하였다.

이 논문에서는 와이블 분포에서 $\beta$ 가 고정되어 있을 때 $\eta$ 가 변화하는 것을 탐지하는 generalized likelihood ratio (Weibull GLR) 관리도 절차를 제안하고자 한다. 특히 $\eta$ 의 감소는 평균수명을 감소시키기 때문에 $\eta$ 가 감소하는 것을 탐지하는 절차를 고려하였다. 일반적으로 CUSUM 관리도는 탐지하고자 하 는 모수의 변화량을 사전에 지정해야 하고, EWMA 관리도는 가중치(weight)를 사전에 지정해야 하는 어려움이 있다. 또한 CUSUM 관리도와 EWMA 관리도의 효율은 사전에 지정하는 관리모수에 많은 영 향을 받는다고 알려져 있다. 즉, CUSUM 관리도는 모형에서 변화를 탐지하고자 하는 모수인 공정모수 
가 미리 지정한 변화량과 유사하게 변화했을 경우 관리도의 효율은 좋지만 그렇지 않은 경우 효율이 떨 어진다는 특징이 있다. 또한 EWMA 관리도는 미리 지정한 가중치가 작은 경우 모수의 작은 변화의 탐 지에는 효율적이지만 큰 변화의 탐지에는 효율이 떨어진다고 알려져 있다. 따라서 두 관리도 모두 사전 에 지정하는 관리모수가 관리도의 성능에 큰 영향을 미칠 수 있기 때문에, 때로는 연구자의 주관적 판단 이 중요한 역할을 하기도 한다 (Reynolds 등, 2013).

그러나 GLR 관리도는 매시점마다 모수의 변화량을 추정하여 사용하기 때문에, 관리한계 외에 추가적으 로 지정해야 할 관리모수가 없다는 장점이 있다. 또한 GLR 관리도는 공정의 변화시점(process changepoint)과 모수 변화량의 추정값을 제공해 주기 때문에 이상원인에 대한 진단(diagnosis)에도 효율적이라 고 알려져 있다. 이 논문에서는 제 1 형 우측중도절단된 와이블 데이터를 모니터링하는 경우 $\eta$ 가 감소하 는 것을 탐지하는 Weibull GLR 관리도 절차를 제안하고, 모의실험(simulation)을 통해 기존에 제안된 관리도와 효율을 비교하여 그 특징을 기술하고 있다.

\section{2. 기존에 제안된 관리도 절차}

\section{1. 조건부 기댓값을 사용한 EWMA 관리도}

Zhang과 Chen (2004)은 Steiner와 MacKay (2001)가 제안한 양쪽 EWMA 관리도의 성능을 향상시 키기 위해 반사벽(reflecting barrier)을 이용하여 공정모수가 증가하는 경우와 감소하는 경우를 각각 탐 지하는 한쪽 $\mathrm{EWMA} \mathrm{CEV} \mathrm{관리도를} \mathrm{제안하였다.} \mathrm{먼저} \mathrm{확률변수} T$ 가 $\operatorname{Weibull}\left(\beta, \eta_{0}\right)$ 를 따른다고 할 때 Zhang과 Chen (2004)은 $Y=\left(T / \eta_{0}\right)^{\beta}$ 로 변수변환을 실시하였고, 이 경우 확률변수 $Y$ 는 평균이 1 인 지 수분포를 따르게 된다. 이때 $Y$ 의 조건부 기댓값(conditional expected value; $\mathrm{CEV}$ )은 다음과 같이 정 의된다.

$$
\mathrm{CE}(Y)=E\left(Y \mid Y>\left(\frac{C}{\eta_{0}}\right)^{\beta}\right)=\left(\frac{C}{\eta_{0}}\right)^{\beta}+1=-\log (\mathrm{pc})+1 .
$$

Zhang과 Chen (2004)은 중도 절단시점이 $C$ 이고 각 시점에서 $n$ 개의 표본을 관측할 때, Weibull $\left(\beta, \eta_{0}\right)$ 를 따르는 수명 $T_{i j}(i=1,2, \ldots, j=1,2, \ldots, n)$ 의 변수변환된 새로운 확률변수 $W_{i j}$ 를 다음과 같이 정의 하였다.

$$
W_{i j}= \begin{cases}\left(\frac{T_{i j}}{\eta_{0}}\right)^{\beta}, & T_{i j} \leq C \\ \mathrm{CE}(Y), & T_{i j}>C .\end{cases}
$$

그들은 시점 $t$ 에서 고정된 $\beta$ 에 대하여 $\eta$ 가 감소하는 것을 탐지하는 한쪽 $\mathrm{EWMA} \mathrm{CEV} \mathrm{관리도의} \mathrm{관리}$ 통계량으로

$$
Q_{t}^{-}=\min \left((1-\lambda) Q_{t-1}^{-}+\lambda \bar{W}_{t}, w_{0}\right), \quad Q_{0}^{-}=w_{0}
$$

을 제안하였다. 여기서 $\bar{W}_{t}=\left(W_{t 1}+W_{t 2}+\cdots+W_{t n}\right) / n, \lambda$ 는 $0<\lambda \leq 1$ 의 범위를 갖는 가중치, 그리 고 $w_{0}$ 는 관리도의 민감도를 향상시키기 위해 사용하는 반사벽이다.

관리상태에서 $W_{t j}$ 의 평균은 1 이기 때문에 Zhang과 Chen (2004)은 $w_{0}=1$ 을 사용하였고, 감소를 탐지 하는 한쪽 $\mathrm{EWMA} \mathrm{CEV}$ 관리도는 미리 설정한 관리한계 $h_{Q}$ 에 대해 $Q_{t}^{-}<h_{Q}$ 인 경우 이상상태라고 판 단한다. 관리한계는 일반적으로 주어진 관리상태에서의 특성을 만족하도록 설정하게 된다. 


\section{2. 우도비에 기초한 CUSUM 관리도}

Dickinson 등 (2014)은 확률변수 $T_{i j}$ 가 $\operatorname{Weibull}\left(\beta, \eta_{0}\right)$ 를 따를 때 중도절단시점 $C$ 에 대하여 다음과 같 이 확률변수를 정의하였다.

$$
T_{i j}=\left\{\begin{array}{ll}
T_{i j}, & T_{i j} \leq C, \\
C, & T_{i j}>C,
\end{array} \quad \delta_{i j}= \begin{cases}1, & T_{i j} \leq C, \\
0, & T_{i j}>C .\end{cases}\right.
$$

$\mathbf{T}_{\mathbf{i}}=\left(T_{i 1}, T_{i 2}, \ldots, T_{i n}\right)$ 라 할 때 $i$ 번째 표본에 대한 우도함수(likelihood function)는

$$
L\left(\beta, \eta \mid \mathbf{T}_{\mathbf{i}}\right)=\prod_{j=1}^{n} f\left(T_{i j} \mid \beta, \eta\right)^{\delta_{i j}}\left[1-F\left(T_{i j} \mid \beta, \eta\right)\right]^{1-\delta_{i j}}
$$

이기 때문에, 로그우도비 통계량 $Z_{i}$ 는 다음과 같이 계산할 수 있다.

$$
\begin{aligned}
Z_{i} & =\log \left(\frac{L\left(\beta, \eta_{1} \mid \mathbf{T}_{\mathbf{i}}\right)}{L\left(\beta, \eta_{0} \mid \mathbf{T}_{\mathbf{i}}\right)}\right) \\
& =X_{i} \beta \log \left(\frac{\eta_{0}}{\eta_{1}}\right)-\sum_{j=1}^{n}\left[\left(\frac{T_{i j}}{\eta_{1}}\right)^{\beta}-\left(\frac{T_{i j}}{\eta_{0}}\right)^{\beta}\right] \\
& =X_{i} \beta \log \left(\frac{1}{1-d}\right)+\left(1-\left(\frac{1}{1-d}\right)^{\beta}\right) \sum_{j=1}^{n}\left(\frac{T_{i j}}{\eta_{0}}\right)^{\beta} \\
& =\left(1-\left(\frac{1}{1-d}\right)^{\beta}\right)\left[\sum_{j=1}^{n}\left(\frac{T_{i j}}{\eta_{0}}\right)^{\beta}-k_{i}\right]
\end{aligned}
$$

여기서 $X_{i}=\sum_{j=1}^{n} \delta_{i j}$ 는 $i$ 번째 표본에서 중도절단시점 $C$ 이전에 고장이 발생하여 실제 고장 자료를 얻 은 갯수를 나타내고, $d(0<d<1)$ 는 $\eta_{1}=(1-d) \eta_{0}$ 에서 척도모수의 감소율을 나타낸다.

Dickinson 등 (2014)은 시점 $t$ 에서 척도모수가 $\eta_{1}=(1-d) \eta_{0}$ 으로 감소하는 것을 탐지하는 한쪽 CUSUM 관리도의 관리통계량을 다음과 같이 제안하였다.

$$
C_{t}^{-}=\min \left(0, C_{t-1}^{-}+\sum_{j=1}^{n}\left(\frac{T_{t j}}{\eta_{0}}\right)^{\beta}-k_{t}\right), \quad C_{0}^{-}=0 .
$$

이때 참고값(reference value) $k_{t}$ 는

$$
k_{t}=-\frac{X_{t} \beta \log \left(\frac{1}{1-d}\right)}{1-\left(\frac{1}{1-d}\right)^{\beta}}
$$

로 표현되며, 한쪽 CUSUM 관리도는 미리 설정한 관리한계 $h_{C}$ 에 대해 $C_{t}^{-}<h_{C}$ 인 경우 이상상태라고 판단한다.

\subsection{BCUSUM 관리도}

Choi와 Lee (2016)는 척도모수가 변화할 경우 중도절단율 pc도 변화하기 때문에, 중도절단되지 않은 표본의 개수를 통계량으로 사용하는 이산형 관리도를 제안하였다. 각 시점에서 $n$ 개의 표본 중 중도절단 되지 않은 표본의 개수 $X_{i}=\sum_{j=1}^{n} \delta_{i j}(i=1,2, \ldots)$ 는 이항분포 $B(n, \mathrm{qc})$ 를 따르는데 이때 $\mathrm{qc}$ 는 비중 
도절단율 $1-\mathrm{qc}$ 를 나타낸다. 식 (1.1)로부터 형상모수 $\beta$ 가 고정되어 있을 때 척도모수 $\eta$ 가 감소할 경 우, 중도절단율 $\mathrm{pc}$ 는 역시 감소하며 따라서 비중도절단율 $\mathrm{qc}$ 는 증가하는 것을 알 수 있다. Choi와 Lee (2016)는 비중도절단율 qc가 증가하는 것을 탐지하기 위해 중도절단되지 않은 표본의 개수 $X_{i}$ 를 통계 량으로 사용하는 이산형 이항 CUSUM (binomial CUSUM; BCUSUM) 관리도를 제안하였다.

시점 $t$ 에서 관리상태의 비중도절단율 $\mathrm{qc}_{0}$ 가 $\mathrm{qc}_{1}$ 으로 증가하는 것을 탐지하는 BCUSUM 관리도의 관리 통계량 $S_{t}^{+}$는 일반적으로 불량률의 증가를 탐지하는 경우와 유사하게

$$
S_{t}^{+}=\max \left(0, S_{t-1}^{+}+X_{t}-k\right), \quad S_{0}^{+}=0
$$

로 정의할 수 있으며, 이때 참고값 $k$ 는

$$
k=\frac{n \log \left(\frac{1-\mathrm{qc}_{0}}{1-\mathrm{qc}_{1}}\right)}{\log \left(\frac{\mathrm{qc}_{1}\left(1-\mathrm{qc}_{0}\right)}{\mathrm{qc}_{0}\left(1-\mathrm{qc}_{1}\right)}\right)}
$$

이다. 여기서 $\mathrm{qc}_{0}=1-\mathrm{pc}_{0}$ 와 $\mathrm{qc}_{1}=1-\mathrm{pc}_{1}$ 은 식 (1.1)의 $\eta$ 에 각각 $\eta_{0}$ 와 $\eta_{1}$ 을 대입해 계산할 수 있다. 한쪽 BCUSUM 관리도는 미리 설정한 관리한계 $h_{S}$ 에 대해 $S_{t}^{+}>h_{S}$ 인 경우 이상상태라고 판단한다.

\section{Weibull GLR 관리도}

Dickinson 등 (2014)이 제안한 CUSUM 관리도와 Choi와 Lee (2016)가 제안한 BCUSUM 관리도는 탐 지하고자 하는 척도모수 $\eta_{1}$ (또는 $d$ ) 값을 미리 지정해 주어야 한다. 일반적으로 CUSUM 관리도는 공 정모수가 미리 지정한 값과 유사하게 변화했을 경우 효율이 매우 좋지만 그렇지 않은 경우 효율이 떨어 질 수 있다고 알려져 있다 (Reynolds와 Lou, 2010; Ryan 등, 2011). 실제 공정에서는 모수에 대한 정보 가 부족한 경우가 많기 때문에 공정모수의 변화량을 예상하기 어려운 경우가 종종 발생할 수 있다. 따라 서 모수의 다양한 변화량에 대해 이를 효율적으로 탐지할 수 있는 관리도 절차가 제안되었는데, 몇 개의 CUSUM 관리도를 병행하여 사용하는 절차와 우도비에 기초한 GLR 관리도 (Reynoldsd 등, 2013) 등 을 그 예로 들 수 있다. 이 논문에서는 제 1 형 우측중도절단된 와이블 데이터를 관측하는 경우 $\eta$ 가 감소 하는 것을 탐지하는 Weibull GLR 관리도 절차를 제안하고자 한다.

중도절단시점이 $C$ 일 때, 확률변수 $T_{i j}, \delta_{i j}$, 그리고 $X_{i}$ 는 2.2 절과 동일하게 정의하였다. 공정이 시점 $\tau$ 까지는 관리상태이며 $\tau+1$ 부터는 이상상태라고 가정하자. 이때 $\tau$ 는 공정의 변화시점이라고 정의한 다. 현재 시점을 $t$ 라 하고 이상상태인 $\tau+1$ 부터 $\eta_{0}$ 가 $\eta_{1}$ 으로 변했을 때 우도함수는

$$
\begin{aligned}
L\left(\tau, \beta, \eta_{1} \mid \mathbf{T}_{\mathbf{1}}, \ldots, \mathbf{T}_{\mathbf{t}}\right)= & \prod_{i=1}^{\tau} \prod_{j=1}^{n} f\left(T_{i j} \mid \beta, \eta_{0}\right)^{\delta_{i j}}\left(1-F\left(T_{i j} \mid \beta, \eta_{0}\right)\right)^{1-\delta_{i j}} \\
& \times \prod_{i=\tau+1}^{t} \prod_{j=1}^{n} f\left(T_{i j} \mid \beta, \eta_{1}\right)^{\delta_{i j}}\left(1-F\left(T_{i j} \mid \beta, \eta_{1}\right)\right)^{1-\delta_{i j}}
\end{aligned}
$$

가 된다. 이때 시점 $t$ 에서 $\eta_{1}$ 의 최대우도추정량(maximum likelihood estimator; MLE) $\hat{\eta}_{1}$ 는 위의 우도 함수를 이용하여 다음과 같이 유도할 수 있다.

$$
\hat{\eta}_{1}=\left[\frac{\sum_{i=\tau+1}^{t} \sum_{j=1}^{n} T_{i j}{ }^{\beta}}{\sum_{i=\tau+1}^{t} X_{i}}\right]^{\frac{1}{\beta}} .
$$


만일 공정에 변화가 발생하지 않은 경우 우도함수는

$$
L\left(\infty, \beta, \eta_{0} \mid \mathbf{T}_{\mathbf{1}}, \ldots, \mathbf{T}_{\mathbf{t}}\right)=\prod_{i=1}^{t} \prod_{j=1}^{n} f\left(T_{i j} \mid \beta, \eta_{0}\right)^{\delta_{i j}}\left(1-F\left(T_{i j} \mid \beta, \eta_{0}\right)\right)^{1-\delta_{i j}}
$$

가 된다. GLR 관리도의 관리통계량 $R_{t}$ 는 다음과 같이 로그우도비의 최댓값으로 정의할 수 있다. 즉,

$$
\begin{aligned}
R_{t} & =\ln \frac{\max _{0 \leq \tau \leq t-1, \eta_{1}} L\left(\tau, \beta, \eta_{1} \mid \mathbf{T}_{\mathbf{1}}, \ldots, \mathbf{T}_{\mathbf{t}}\right)}{L\left(\infty, \beta, \eta_{0} \mid \mathbf{T}_{\mathbf{1}}, \ldots, \mathbf{T}_{\mathbf{t}}\right)} \\
& =\ln \frac{\max _{0 \leq \tau \leq t-1, \eta_{1}} \prod_{i=\tau+1}^{t} \prod_{j=1}^{n} f\left(T_{i j} \mid \beta, \eta_{1}\right)^{\delta_{i j}}\left(1-F\left(T_{i j} \mid \beta, \eta_{1}\right)\right)^{1-\delta_{i j}}}{\prod_{i=\tau+1}^{t} \prod_{j=1}^{n} f\left(T_{i j} \mid \beta, \eta_{0}\right)^{\delta_{i j}}\left(1-F\left(T_{i j} \mid \beta, \eta_{0}\right)\right)^{1-\delta_{i j}}} \\
& =\max _{0 \leq \tau \leq t-1}\left[\beta \log \left(\frac{\eta_{0}}{\hat{\eta}_{1}}\right) \sum_{i=\tau+1}^{t} X_{i}+\sum_{i=\tau+1}^{t} \sum_{j=1}^{n}\left(\left(\frac{T_{i j}}{\eta_{0}}\right)^{\beta}-\left(\frac{T_{i j}}{\hat{\eta}_{1}}\right)^{\beta}\right)\right]
\end{aligned}
$$

로 표현할 수 있다. Weibull GLR 관리도 절차는 미리 설정한 관리한계 $h_{\mathrm{GLR}}$ 에 대해 $R_{t} \geq h_{\mathrm{GLR}}$ 인 경 우 이상상태라고 판단한다. 일반적으로 CUSUM 관리도와 EWMA 관리도에서는 Markov 연쇄를 이용 하여 주어진 관리상태에서의 특성을 만족하는 관리한계를 찾을 수 있지만, GLR 관리도의 관리통계량은 Markov 연쇄 조건을 만족하지 않기 때문에 모의실험을 통하여 관리한계 $h_{\mathrm{GLR}}$ 을 설정할 수 있다.

$\mathrm{GLR}$ 관리도의 관리통계량 $R_{t}$ 는 매 시점마다 $\tau$ 를 $0 \leq \tau \leq t-1$ 의 범위에 대해 최댓값을 찾는다. 따라 서 모의실험에서 $t$ 가 커짐에 따라 계산량이 많아져 관리통계량을 구하는데 많은 시간이 소요될 수 있기 때문에, 윈도우 크기 (window size) $m$ 을 사전에 설정하여 $\tau$ 를 축소된 범위인 $t-m \leq \tau \leq t-1$ 에서 최 댓값을 찾는 방법도 있다. 그러나 이 논문에서는 윈도우 크기를 사용하지 않고 모든 범위에서 최댓값을 구하는 방법을 사용하였다.

GLR 관리도는 CUSUM 관리도와는 다르게 사전에 이상상태의 모수값을 지정하지 않아도 되며, 이상신 호가 발생한 후 이상원인에 대한 추가적인 정보를 제공한다는 장점이 있다. 즉, GLR 관리도는 이상신 호가 발생한 시점에서 계산된 $\hat{\tau}$ 와 $\hat{\eta}_{1}$ 을 제공해주기 때문에, 추정된 공정의 변화시점과 모수의 변화량은 이상원인에 대한 공정 진단에 유용하게 사용할 수 있다.

다음으로 Weibull GLR 관리도에 대한 하나의 예를 살펴보고자 한다. Figure 3.1 은 $\beta=1, \eta_{0}=1$, $\eta_{1}=0.85, \mathrm{pc}=0.15, \tau=50$ 일 때 모의실험을 한 번 실시하여 작성한 관리도이다. 즉, 시점 50 까 지는 $\eta=\eta_{0}=1$ 이고 시점 51 부터는 $\eta=\eta_{1}=0.85$ 이며 중도절단율은 $15 \%$ 인 경우이다. 관리한계 $h_{\mathrm{GLR}}=5.50$ 은 $\mathrm{ARL}_{0} \approx 370$ 을 만족하는 값으로 가로선으로 표시하였다. 이 관리도에서 시점 57 번째 관리통계량인 $R_{57}$ 은 6.2191 로서 최초로 관리한계를 벗어났기 때문에 이때 이상신호가 발생했으며, 이 시점에서 추정된 $\hat{\tau}$ 은 50 으로 실제 변화가 일어난 $\tau=50$ 과 동일한 값이었고 $\hat{\eta}_{1}$ 은 0.87 로 실제 값인 $\eta_{1}=0.85$ 와 상당히 유사한 값임을 알 수 있다.

\section{4. 모의실험}

관리도의 성능을 평가하는 척도로 평균런길이(average run length; ARL)가 일반적으로 사용된다. 먼 저 런길이(run length; RL)는 공정이 시작하고 이상신호가 발생할 때까지 추출한 표본의 수를 나타 내는데, 관리상태에서의 런길이는 오경보(false alarm)가 발생할 때까지 추출한 표본의 수가 되고, 이 


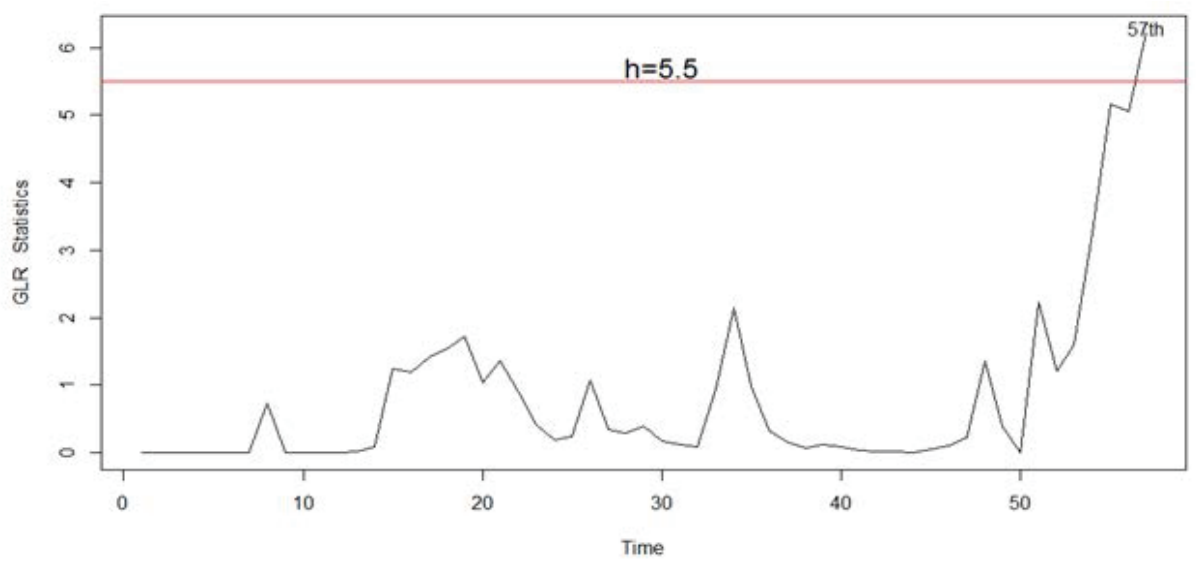

Figure 3.1. Example plot of the Weibull GLR chart when $\beta=1, \eta_{0}=1, \eta_{1}=0.85$, pc $=0.15$, and $\tau=50$.

상상태에서의 런길이는 이상원인이 발생한 후 이상신호가 발생할 때까지 추출한 표본의 수가 된다. 평균런길이는 런길이의 기댓값이 된다. 일반적으로 관리도의 성능을 비교할 때 관리상태의 평균런길 이 $\left(\mathrm{ARL}_{0}\right)$ 는 일정한 값을 유지하도록 관리한계를 설정한 후 이상상태의 평균런길이 $\left(\mathrm{ARL}_{1}\right)$ 를 계산하여 이를 비교하고 있는데, 이 논문에서도 이와 같은 방법을 사용하였다.

Dickinson 등 (2014)은 $\beta$ 가 고정되고 $\eta$ 가 감소하는 경우 그들이 제안한 한쪽 CUSUM 관리도가 Zhang과 Chen (2004)이 제안한 한쪽 EWMA CEV 관리도보다 효율이 우수함을 보였다. 따라서 이 논문은 Dickinson 등 (2014)이 제안한 CUSUM 관리도와 Choi와 Lee (2016)가 BCUSM 관리도를 비 교의 기준으로 삼았고, 이 논문의 모의실험은 그들이 모의실험에서 설정한 가정들을 그대로 사용하였다. 즉, $\beta=0.5,1,3,5$, 중도절단율 $\mathrm{pc}$ 는 $15 \%, 50 \%, 95 \%$ 를 고려하였다. 표본크기 $n$ 은 5 로 설정하였고, 추가적으로 표본크기의 변화에 따른 성능을 측정하기 위해 $\beta=3$ 일 때 $n=3,5,10$ 인 경우에 대해 모의 실험을 실시하였다. 관리한계인 $h_{C}, h_{S}$, 그리고 $h_{\mathrm{GLR}}$ 은 $\mathrm{ARL}_{0} \approx 370$ 을 만족하도록 설정했으며, 공정 의 변화시점은 $\tau=50$ 으로 설정하였다. Weibull GLR 관리도의 $\mathrm{ARL}$ 을 계산하기 위해 각 경우에 대해 10,000 번씩 반복을 하였고, Dickinson 등 (2014)의 CUSUM 관리도와 Choi와 Lee (2016)의 BCUSM 관리도의 경우에는 그들의 논문에 있는 결과를 그대로 사용하였다.

\section{1. 형상모수 $\beta$ 와 중도절단율 $\mathbf{p c}$ 의 변화에 따른 관리도의 성능 비교}

Table 4.1부터 Table 4.4 는 $n=5$ 과 $\eta_{0}=1$ 로 고정되어 있을 때, $\beta=0.5,1,3,5$ 로 변하는 경우 에 대한 평균런길이 $\left(\mathrm{ARL}_{1}\right)$ 를 비교한 결과이다. CUSUM 관리도와 BCUSUM 관리도는 사전에 변화를 탐지하고자 하는 이상상태의 척도모수인 $\eta_{1}$ 값을 지정해야 하는데, 기존 연구인 Dickinson 등 (2014)과 Choi와 Lee (2016)의 경우와 동일하게 $\beta=0.5$ 일 때는 $\eta_{1}=0.65, \beta=1$ 일 때는 $\eta_{1}=0.70, \beta=3$ 일 때 는 $\eta_{1}=0.80$, 그리고 $\beta=5$ 일 때는 $\eta_{1}=0.85$ 로 설정하였다. 비교를 용이하게 하기 위해 $\eta$ 의 각 변화 에 대해 $\mathrm{ARL}_{1}$ 이 가장 작은 값을 진하게 표시하였다.

$\beta=0.5$ 인 Table 4.1 을 보면, 중도절단율 $\mathrm{pc}$ 가 낮은 경우 $(\mathrm{pc}=0.5)$ CUSUM 관리도의 성능이 가장 좋 으며, $\mathrm{pc}$ 가 높아질수록 BCUSUM 관리도의 성능이 좋아짐을 알 수 있다. $\mathrm{pc}=0.95$ 인 경우에는 $\eta$ 의 모든 변화에 대해 BCUSUM 관리도의 성능이 가장 좋게 나타났다. $\beta=0.5$ 인 경우 $\mathrm{pc}$ 에 상관 없이 Weibull GLR 관리도의 성능이 CUSUM 관리도와 BCUSUM 관리도에 비해 좋지 않은 것을 확인할 수 
Table 4.1. Comparison of the $\mathrm{ARL}_{1}$ values for $\beta=0.5, \eta_{0}=1, n=5$ with $\mathrm{ARL}_{0} \approx 370$

\begin{tabular}{|c|c|c|c|c|c|c|}
\hline $\mathrm{pc}$ & $\eta_{1}$ & $\begin{array}{c}\text { CUSUM } \\
h_{C}=-13.623\end{array}$ & $\begin{array}{c}\text { BCUSUM } \\
h_{S}=5.905\end{array}$ & $\begin{array}{c}\text { GLR } \\
h_{\mathrm{GLR}}=5.48\end{array}$ & $\hat{\tau}$ & $\hat{\eta}_{1}$ \\
\hline \multirow{12}{*}{0.15} & 0.95 & 220.75 & 221.27 & 268.78 & 304.2 & 0.20 \\
\hline & 0.90 & 140.58 & 146.02 & 189.55 & 212.7 & 0.26 \\
\hline & 0.85 & 91.98 & 99.52 & 127.24 & 141.1 & 0.32 \\
\hline & 0.80 & 62.16 & 70.32 & 89.64 & 103.0 & 0.36 \\
\hline & 0.75 & 44.23 & 51.23 & 62.63 & 79.3 & 0.39 \\
\hline & 0.70 & 32.55 & 39.01 & 45.89 & 67.7 & 0.39 \\
\hline & 0.65 & 24.84 & 30.45 & 34.12 & 60.1 & 0.38 \\
\hline & 0.60 & 19.61 & 24.67 & 25.94 & 56.1 & 0.36 \\
\hline & 0.55 & 15.95 & 20.49 & 20.25 & 53.3 & 0.35 \\
\hline & 0.50 & 13.27 & 17.32 & 16.19 & 51.6 & 0.33 \\
\hline & 0.45 & 11.18 & 14.92 & 12.69 & 50.6 & 0.31 \\
\hline & 0.40 & 9.52 & 13.13 & 10.28 & 50.1 & 0.28 \\
\hline $\mathrm{pc}$ & $\eta_{1}$ & $\begin{array}{c}\text { CUSUM } \\
h_{C}=-12.125\end{array}$ & $\begin{array}{c}\text { BCUSUM } \\
h_{S}=9.304\end{array}$ & $\begin{array}{c}\text { GLR } \\
h_{\mathrm{GLR}}=5.32\end{array}$ & $\hat{\tau}$ & $\hat{\eta}_{1}$ \\
\hline \multirow{12}{*}{0.5} & 0.95 & 234.77 & 227.48 & 286.08 & 325.1 & 0.15 \\
\hline & 0.90 & 160.11 & 154.78 & 214.63 & 243.3 & 0.20 \\
\hline & 0.85 & 109.88 & 107.68 & 158.07 & 179.8 & 0.24 \\
\hline & 0.80 & 87.51 & 76.81 & 115.44 & 132.7 & 0.28 \\
\hline & 0.75 & 57.01 & 55.77 & 85.57 & 101.6 & 0.31 \\
\hline & 0.70 & 42.48 & 41.92 & 62.11 & 81.8 & 0.32 \\
\hline & 0.65 & 32.70 & 32.38 & 47.23 & 70.1 & 0.33 \\
\hline & 0.60 & 25.75 & 25.62 & 36.03 & 62.6 & 0.32 \\
\hline & 0.55 & 20.80 & 20.70 & 27.64 & 57.9 & 0.31 \\
\hline & 0.50 & 16.97 & 17.00 & 21.57 & 55.0 & 0.29 \\
\hline & 0.45 & 14.15 & 14.13 & 17.02 & 52.8 & 0.28 \\
\hline & 0.40 & 11.84 & 11.96 & 13.26 & 51.3 & 0.25 \\
\hline $\mathrm{pc}$ & $\eta_{1}$ & $\begin{array}{c}\text { CUSUM } \\
h_{C}=-6.113\end{array}$ & $\begin{array}{c}\text { BCUSUM } \\
h_{S}=6.597\end{array}$ & $\begin{array}{c}\text { GLR } \\
h_{\mathrm{GLR}}=4.62\end{array}$ & $\hat{\tau}$ & $\hat{\eta}_{1}$ \\
\hline \multirow{12}{*}{0.95} & 0.95 & 291.78 & 276.63 & 319.87 & 362.1 & 0.04 \\
\hline & 0.90 & 246.02 & 232.44 & 292.28 & 333.3 & 0.04 \\
\hline & 0.85 & 205.24 & 196.01 & 260.07 & 299.3 & 0.05 \\
\hline & 0.80 & 175.13 & 166.57 & 232.68 & 270.1 & 0.05 \\
\hline & 0.75 & 146.90 & 140.22 & 197.73 & 233.2 & 0.06 \\
\hline & 0.70 & 123.51 & 117.76 & 169.28 & 201.9 & 0.06 \\
\hline & 0.65 & 104.87 & 99.22 & 147.57 & 177.7 & 0.07 \\
\hline & 0.60 & 87.48 & 83.61 & 122.20 & 151.3 & 0.08 \\
\hline & 0.55 & 74.75 & 70.89 & 104.61 & 132.6 & 0.08 \\
\hline & 0.50 & 62.40 & 59.92 & 87.05 & 114.6 & 0.09 \\
\hline & 0.45 & 52.79 & 50.09 & 70.53 & 98.6 & 0.09 \\
\hline & 0.40 & 44.38 & 42.36 & 57.49 & 86.7 & 0.09 \\
\hline
\end{tabular}

The CUSUM and BCUSUM charts are designed to detect $\eta_{1}=0.65$.

$\mathrm{ARL}=$ average run length; CUSUM $=$ cumulative sum; BCUSUM $=$ binomial CUSUM; GLR = generalized likelihood ratio. 
Table 4.2. Comparison of the $\mathrm{ARL}_{1}$ values for $\beta=1, \eta_{0}=1, n=5$ with $\mathrm{ARL}_{0} \approx 370$

\begin{tabular}{|c|c|c|c|c|c|c|}
\hline $\mathrm{pc}$ & $\eta_{1}$ & $\begin{array}{c}\text { CUSUM } \\
h_{C}=-9.141\end{array}$ & $\begin{array}{c}\text { BCUSUM } \\
h_{S}=4.199\end{array}$ & $\begin{array}{c}\text { GLR } \\
h_{\mathrm{GLR}}=5.49\end{array}$ & $\hat{\tau}$ & $\hat{\eta}_{1}$ \\
\hline \multirow{12}{*}{0.15} & 0.95 & 168.04 & 178.63 & 193.98 & 216.3 & 0.45 \\
\hline & 0.90 & 84.32 & 93.89 & 96.13 & 109.3 & 0.54 \\
\hline & 0.85 & 45.34 & 53.48 & 52.81 & 72.4 & 0.58 \\
\hline & 0.80 & 26.81 & 33.59 & 32.41 & 59.2 & 0.58 \\
\hline & 0.75 & 17.49 & 23.11 & 21.51 & 53.9 & 0.56 \\
\hline & 0.70 & 12.60 & 17.25 & 15.29 & 51.4 & 0.54 \\
\hline & 0.65 & 9.60 & 13.79 & 11.38 & 50.3 & 0.52 \\
\hline & 0.60 & 7.69 & 11.56 & 8.56 & 49.8 & 0.48 \\
\hline & 0.55 & 6.38 & 10.06 & 6.73 & 49.5 & 0.45 \\
\hline & 0.50 & 5.42 & 9.06 & 5.33 & 49.5 & 0.42 \\
\hline & 0.45 & 4.73 & 8.35 & 4.39 & 49.5 & 0.39 \\
\hline & 0.40 & 4.20 & 7.86 & 3.60 & 49.5 & 0.35 \\
\hline $\mathrm{pc}$ & $\eta_{1}$ & $\begin{array}{c}\text { CUSUM } \\
h_{C}=-4.421\end{array}$ & $\begin{array}{c}\text { BCUSUM } \\
h_{S}=6.795\end{array}$ & $\begin{array}{c}\text { GLR } \\
h_{\mathrm{GLR}}=5.32\end{array}$ & $\hat{\tau}$ & $\hat{\eta}_{1}$ \\
\hline \multirow{12}{*}{0.5} & 0.95 & 186.42 & 180.61 & 223.24 & 252.1 & 0.38 \\
\hline & 0.90 & 100.08 & 97.59 & 124.56 & 140.5 & 0.47 \\
\hline & 0.85 & 57.21 & 56.33 & 72.43 & 89.9 & 0.51 \\
\hline & 0.80 & 35.19 & 34.79 & 44.17 & 68.5 & 0.52 \\
\hline & 0.75 & 23.24 & 23.35 & 29.56 & 59.0 & 0.52 \\
\hline & 0.70 & 16.53 & 16.69 & 20.63 & 54.1 & 0.51 \\
\hline & 0.65 & 12.40 & 12.59 & 14.72 & 52.1 & 0.48 \\
\hline & 0.60 & 9.66 & 9.90 & 11.15 & 50.8 & 0.46 \\
\hline & 0.55 & 7.81 & 8.02 & 8.41 & 50.1 & 0.43 \\
\hline & 0.50 & 6.44 & 6.68 & 6.53 & 49.8 & 0.40 \\
\hline & 0.45 & 5.44 & 5.65 & 5.14 & 49.5 & 0.37 \\
\hline & 0.40 & 4.62 & 4.89 & 4.11 & 49.5 & 0.34 \\
\hline $\mathrm{pc}$ & $\eta_{1}$ & $\begin{array}{c}\text { CUSUM } \\
h_{C}=-3.891\end{array}$ & $\begin{array}{c}\text { BCUSUM } \\
h_{S}=5.675\end{array}$ & $\begin{array}{c}\text { GLR } \\
h_{\mathrm{GLR}}=4.60\end{array}$ & $\hat{\tau}$ & $\hat{\eta}_{1}$ \\
\hline \multirow{12}{*}{0.95} & 0.95 & 257.92 & 244.50 & 298.00 & 339.3 & 0.16 \\
\hline & 0.90 & 189.84 & 181.36 & 239.07 & 276.9 & 0.18 \\
\hline & 0.85 & 141.69 & 133.16 & 186.06 & 220.7 & 0.19 \\
\hline & 0.80 & 106.43 & 100.55 & 144.23 & 174.3 & 0.21 \\
\hline & 0.75 & 80.70 & 76.14 & 111.01 & 139.2 & 0.23 \\
\hline & 0.70 & 62.17 & 58.83 & 84.94 & 112.6 & 0.24 \\
\hline & 0.65 & 48.45 & 45.80 & 66.05 & 94.3 & 0.25 \\
\hline & 0.60 & 38.30 & 36.20 & 50.07 & 80.8 & 0.25 \\
\hline & 0.55 & 30.51 & 28.80 & 38.89 & 71.1 & 0.25 \\
\hline & 0.50 & 24.51 & 23.29 & 29.82 & 64.1 & 0.24 \\
\hline & 0.45 & 19.86 & 18.70 & 22.77 & 59.4 & 0.23 \\
\hline & 0.40 & 16.01 & 15.13 & 16.84 & 55.6 & 0.22 \\
\hline
\end{tabular}

The CUSUM and BCUSUM charts are designed to detect $\eta_{1}=0.70$.

$\mathrm{ARL}=$ average run length; CUSUM = cumulative sum; BCUSUM = binomial CUSUM; GLR = generalized likelihood ratio. 
Table 4.3. Comparison of the $\mathrm{ARL}_{1}$ values for $\beta=3, \eta_{0}=1, n=5$ with $\mathrm{ARL}_{0} \approx 370$

\begin{tabular}{|c|c|c|c|c|c|c|}
\hline $\mathrm{pc}$ & $\eta_{1}$ & $\begin{array}{c}\text { CUSUM } \\
h_{C}=-4.474\end{array}$ & $\begin{array}{c}\text { BCUSUM } \\
h_{S}=2.244\end{array}$ & $\begin{array}{c}\text { GLR } \\
h_{\mathrm{GLR}}=5.48\end{array}$ & $\hat{\tau}$ & $\hat{\eta}_{1}$ \\
\hline \multirow{12}{*}{0.15} & 0.95 & 77.15 & 78.89 & 56.98 & 75.7 & 0.81 \\
\hline & 0.90 & 21.37 & 25.71 & 18.55 & 52.6 & 0.81 \\
\hline & 0.85 & 8.92 & 12.86 & 9.24 & 50.0 & 0.78 \\
\hline & 0.80 & 5.19 & 8.70 & 5.66 & 49.5 & 0.74 \\
\hline & 0.75 & 3.70 & 7.03 & 3.91 & 49.5 & 0.70 \\
\hline & 0.70 & 2.96 & 6.35 & 2.90 & 49.6 & 0.66 \\
\hline & 0.65 & 2.52 & 6.10 & 2.28 & 49.6 & 0.62 \\
\hline & 0.60 & 2.21 & 6.02 & 1.87 & 49.7 & 0.57 \\
\hline & 0.55 & 2.00 & 6.01 & 1.57 & 49.8 & 0.53 \\
\hline & 0.50 & 1.92 & 6.01 & 1.31 & 49.8 & 0.48 \\
\hline & 0.45 & 1.89 & 6.01 & 1.10 & 49.9 & 0.44 \\
\hline & 0.40 & 1.88 & 6.01 & 1.01 & 49.9 & 0.39 \\
\hline $\mathrm{pc}$ & $\eta_{1}$ & $\begin{array}{c}\text { CUSUM } \\
h_{C}=-4.400\end{array}$ & $\begin{array}{c}\text { BCUSUM } \\
h_{S}=4.084\end{array}$ & $\begin{array}{c}\text { GLR } \\
h_{\mathrm{GLR}}=5.32\end{array}$ & $\hat{\tau}$ & $\hat{\eta}_{1}$ \\
\hline \multirow{12}{*}{0.5} & 0.95 & 87.02 & 85.56 & 76.89 & 94.9 & 0.77 \\
\hline & 0.90 & 26.38 & 26.81 & 25.45 & 56.9 & 0.78 \\
\hline & 0.85 & 11.22 & 11.60 & 12.05 & 51.2 & 0.76 \\
\hline & 0.80 & 6.33 & 6.67 & 6.95 & 49.9 & 0.73 \\
\hline & 0.75 & 4.27 & 4.62 & 4.57 & 49.5 & 0.69 \\
\hline & 0.70 & 3.21 & 3.60 & 3.17 & 49.6 & 0.65 \\
\hline & 0.65 & 2.60 & 3.06 & 2.41 & 49.6 & 0.61 \\
\hline & 0.60 & 2.22 & 2.77 & 1.89 & 49.7 & 0.57 \\
\hline & 0.55 & 1.99 & 2.65 & 1.55 & 49.8 & 0.52 \\
\hline & 0.50 & 1.90 & 2.60 & 1.29 & 49.8 & 0.48 \\
\hline & 0.45 & 1.87 & 2.60 & 1.08 & 49.9 & 0.43 \\
\hline & 0.40 & 1.86 & 2.60 & 1.00 & 49.9 & 0.39 \\
\hline $\mathrm{pc}$ & $\eta_{1}$ & $\begin{array}{c}\text { CUSUM } \\
h_{C}=-3.461\end{array}$ & $\begin{array}{c}\text { BCUSUM } \\
h_{S}=4.3094\end{array}$ & $\begin{array}{c}\text { GLR } \\
h_{\mathrm{GLR}}=4.62\end{array}$ & $\hat{\tau}$ & $\hat{\eta}_{1}$ \\
\hline \multirow{12}{*}{0.95} & 0.95 & 169.31 & 155.20 & 196.02 & 231.2 & 0.54 \\
\hline & 0.90 & 80.82 & 76.40 & 100.73 & 128.4 & 0.58 \\
\hline & 0.85 & 43.53 & 41.49 & 55.01 & 84.6 & 0.59 \\
\hline & 0.80 & 26.15 & 24.77 & 31.24 & 64.8 & 0.59 \\
\hline & 0.75 & 16.82 & 16.00 & 19.39 & 57.0 & 0.58 \\
\hline & 0.70 & 11.52 & 11.00 & 12.34 & 53.3 & 0.56 \\
\hline & 0.65 & 8.25 & 7.88 & 8.50 & 51.5 & 0.54 \\
\hline & 0.60 & 6.08 & 5.84 & 5.67 & 50.4 & 0.52 \\
\hline & 0.55 & 4.55 & 4.38 & 3.93 & 49.8 & 0.49 \\
\hline & 0.50 & 3.46 & 3.36 & 2.81 & 49.7 & 0.46 \\
\hline & 0.45 & 2.67 & 2.62 & 2.02 & 49.7 & 0.42 \\
\hline & 0.40 & 2.09 & 2.08 & 1.48 & 49.8 & 0.38 \\
\hline
\end{tabular}

The CUSUM and BCUSUM charts are designed to detect $\eta_{1}=0.80$.

$\mathrm{ARL}=$ average run length; CUSUM $=$ cumulative sum; BCUSUM $=$ binomial CUSUM; GLR $=$ generalized likelihood ratio. 
Table 4.4. Comparison of the $\mathrm{ARL}_{1}$ values for $\beta=5, \eta_{0}=1, n=5$ with $\mathrm{ARL}_{0} \approx 370$

\begin{tabular}{|c|c|c|c|c|c|c|}
\hline $\mathrm{pc}$ & $\eta_{1}$ & $\begin{array}{c}\text { CUSUM } \\
h_{C}=-2.200\end{array}$ & $\begin{array}{c}\text { BCUSUM } \\
h_{S}=1.763\end{array}$ & $\begin{array}{c}\text { GLR } \\
h_{\mathrm{GLR}}=5.50\end{array}$ & $\hat{\tau}$ & $\hat{\eta}_{1}$ \\
\hline \multirow{12}{*}{0.15} & 0.95 & 40.30 & 40.90 & 25.93 & 55.8 & 0.88 \\
\hline & 0.90 & 8.67 & 12.21 & 8.27 & 49.7 & 0.85 \\
\hline & 0.85 & 3.98 & 7.44 & 4.27 & 49.5 & 0.81 \\
\hline & 0.80 & 2.67 & 6.31 & 2.76 & 49.5 & 0.77 \\
\hline & 0.75 & 2.14 & 6.09 & 2.02 & 49.7 & 0.73 \\
\hline & 0.70 & 1.92 & 6.06 & 1.58 & 49.8 & 0.68 \\
\hline & 0.65 & 1.87 & 6.06 & 1.25 & 49.8 & 0.63 \\
\hline & 0.60 & 1.85 & 6.06 & 1.04 & 49.9 & 0.59 \\
\hline & 0.55 & 1.83 & 6.06 & 1.00 & 49.9 & 0.54 \\
\hline & 0.50 & 1.81 & 6.06 & 1.00 & 49.9 & 0.49 \\
\hline & 0.45 & 1.80 & 6.05 & 1.00 & 49.9 & 0.44 \\
\hline & 0.40 & 1.80 & 6.05 & 1.00 & 49.9 & 0.39 \\
\hline $\mathrm{pc}$ & $\eta_{1}$ & $\begin{array}{c}\text { CUSUM } \\
h_{C}=-2.158\end{array}$ & $\begin{array}{c}\text { BCUSUM } \\
h_{S}=3.352\end{array}$ & $\begin{array}{c}\text { GLR } \\
h_{\mathrm{GLR}}=5.31\end{array}$ & $\hat{\tau}$ & $\hat{\eta}_{1}$ \\
\hline \multirow{12}{*}{0.5} & 0.95 & 47.05 & 49.34 & 35.19 & 62.1 & 0.86 \\
\hline & 0.90 & 10.74 & 11.54 & 10.49 & 50.8 & 0.84 \\
\hline & 0.85 & 4.66 & 5.16 & 4.98 & 49.6 & 0.80 \\
\hline & 0.80 & 2.92 & 3.30 & 2.99 & 49.5 & 0.77 \\
\hline & 0.75 & 2.21 & 2.47 & 2.05 & 49.7 & 0.72 \\
\hline & 0.70 & 1.93 & 2.09 & 1.55 & 49.8 & 0.68 \\
\hline & 0.65 & 1.85 & 1.96 & 1.22 & 49.8 & 0.63 \\
\hline & 0.60 & 1.82 & 1.94 & 1.03 & 49.9 & 0.59 \\
\hline & 0.55 & 1.79 & 1.94 & 1.00 & 49.9 & 0.54 \\
\hline & 0.50 & 1.77 & 1.94 & 1.00 & 49.9 & 0.49 \\
\hline & 0.45 & 1.77 & 1.94 & 1.00 & 49.9 & 0.44 \\
\hline & 0.40 & 1.76 & 1.94 & 1.00 & 49.9 & 0.39 \\
\hline $\mathrm{pc}$ & $\eta_{1}$ & $\begin{array}{c}\text { CUSUM } \\
h_{C}=-1.820\end{array}$ & $\begin{array}{c}\text { BCUSUM } \\
h_{S}=3.873\end{array}$ & $\begin{array}{c}\text { GLR } \\
h_{\mathrm{GLR}}=4.61\end{array}$ & $\hat{\tau}$ & $\hat{\eta}_{1}$ \\
\hline \multirow{12}{*}{0.95} & 0.95 & 110.12 & 105.49 & 125.67 & 155.6 & 0.70 \\
\hline & 0.90 & 40.79 & 39.23 & 48.74 & 79.0 & 0.73 \\
\hline & 0.85 & 19.09 & 18.28 & 21.56 & 58.4 & 0.72 \\
\hline & 0.80 & 10.57 & 10.18 & 11.54 & 52.8 & 0.70 \\
\hline & 0.75 & 6.62 & 6.37 & 6.55 & 50.7 & 0.68 \\
\hline & 0.70 & 4.40 & 4.28 & 4.05 & 49.9 & 0.65 \\
\hline & 0.65 & 3.10 & 3.03 & 2.57 & 49.8 & 0.61 \\
\hline & 0.60 & 2.27 & 2.22 & 1.75 & 49.7 & 0.58 \\
\hline & 0.55 & 1.75 & 1.73 & 1.26 & 49.8 & 0.54 \\
\hline & 0.50 & 1.42 & 1.41 & 1.05 & 49.9 & 0.49 \\
\hline & 0.45 & 1.14 & 1.14 & 1.00 & 49.9 & 0.44 \\
\hline & 0.40 & 1.01 & 1.01 & 1.00 & 49.9 & 0.39 \\
\hline
\end{tabular}

The CUSUM and BCUSUM charts are designed to detect $\eta_{1}=0.85$.

$\mathrm{ARL}=$ average run length; CUSUM = cumulative sum; BCUSUM = binomial CUSUM; GLR = generalized likelihood ratio. 
있다. 그 이유를 살펴보면, $\eta$ 의 변화가 큰 경우 $\tau$ 의 추정이 실제값인 $\tau=50$ 과 유사한 값을 갖지만 $\eta$ 의 변화가 작거나 또는 중도절단율 $\mathrm{pc}$ 가 높아지는 경우 추정의 정도가 매우 나빠진다. 또한 $\mathrm{pc}$ 가 높아질수 록 $\eta_{1}$ 의 추정이 불정확해져서 이로 인하여 GLR 관리도의 성능이 나빠지는 것으로 판단된다.

Table 4.2 는 $\beta=1$ 인 경우에 대한 결과이다. $\beta=0.5$ 일 때와 전반적으로 유사한 경향을 가지고 있지 만, pc가 0.15 와 0.5 인 경우 $\eta$ 의 아주 큰 변화에 대해 Weibull GLR 관리도가 우수한 성능을 나타냈다. $\mathrm{pc}=0.95$ 일 경우에는 여전히 Weibull GLR 관리도의 성능이 CUSUM 관리도와 BCUSUM 관리도에 비해 좋지 않게 나타났으며, 이 경우 BCUSUM 관리도의 성능이 가장 좋게 나타났다. 그러나 $\hat{\tau}$ 과 $\hat{\eta}_{1}$ 을 살펴보면, $\beta=0.5$ 인 경우에 비해 추정의 정도가 조금 좋아진 것을 알 수 있고, 따라서 Weibull GLR 관 리도의 성능은 $\beta$ 와 연관이 있다고 생각할 수 있다.

Table 4.3과 Table 4.4는 각각 $\beta=3$ 과 5 에 대한 결과이다. $\beta$ 가 커질수록 Weibull GLR 관리도의 성 능이 가장 좋은 경우가 많이 발생하며, $\mathrm{pc}=0.15$ 와 0.5 인 경우 $\eta$ 가 CUSUM과 BCUSUM에서 사전에 설정한 $\eta_{1}$ 과 유사하게 변화한 경우를 제외하고 Weibull GLR 관리도의 성능이 가장 좋은 것을 알 수 있 다. $\mathrm{pc}=0.95$ 일 때에도 $\eta$ 가 크게 변하는 경우에는 Weibull GLR 관리도의 성능이 가장 좋게 나타났다. 또한 $\eta_{1}=0.95$ 인 경우를 제외하고는 $\tau$ 와 $\eta_{1}$ 의 추정의 정도가 많이 향상된 것을 확인할 수 있다.

Table 4.1 에서 Table 4.4 의 결과를 종합적으로 살펴볼 때, 형상모수 $\beta$ 가 큰 값을 갖고 중도절단율 $\mathrm{pc}$ 가 아주 높지 않은 경우 기존의 관리도에 비해 Weibull GLR 관리도의 성능이 우수함을 알 수 있다.

\section{2. 표본크기 $n$ 과 중도절단율 $\mathrm{pc}$ 의 변화에 따른 관리도의 성능 비교}

Table 4.5부터 Table 4.7 은 $\beta=3$ 으로 고정시켰을 때 중도절단율 $\mathrm{pc}=0.15,0.5,0.95$ 에 대하여 $n=3$, 5,10 으로 변하는 경우 각 관리도의 성능을 비교한 결과이다. $\mathrm{pc}=0.15$ 인 Table 4.5 를 살펴보면, 전반 적으로 Weibull GLR 관리도가 표본크기 $n$ 에 상관 없이 $\eta$ 의 큰 변화와 작은 변화를 빨리 탐지함을 알 수 있다. 특히, $n$ 이 증가할수록 $\tau$ 와 $\eta$ 의 추정이 정확해지면서 $\eta$ 의 거의 모든 변화에 대해 성능이 향상 되는 것을 확인할 수 있다.

$\mathrm{pc}=0.5$ 인 Table 4.6 에서는 $n=3$ 인 경우 $\eta$ 의 큰 변화에 대해서만 Weibull GLR 관리도의 성능이 좋 았지만, $n=5$ 와 10 으로 증가할 경우 Table 4.5 와 동일하게 $\eta$ 의 대부분의 변화에 대해 가장 우수한 성 능을 나타내는 것을 확인할 수 있다.

마지막으로 $\mathrm{pc}=0.95$ 인 Table 4.7 을 살펴보면, $n$ 이 증가해도 $\eta$ 의 큰 변화에 대해서만 Weibull GLR 관리도의 성능이 가장 좋았으며 그 외의 변화에 대해선 BCUSUM 관리도의 성능이 가장 좋은 것을 알 수 있다.

\section{5. 결론}

제품의 수명시험은 실제 산업현장에서 빈번하게 사용되며 여러 가지 제약으로 인하여 자료의 중도절단 이 자주 발생하는데, 이 논문은 제 1 형 우측중도절단이 있는 와이블 수명자료에 대해 척도모수의 변화를 탐지하는 Weibull GLR 관리도를 제안하였다. Weibull GLR 관리도는 이상상태의 모수값을 지정할 필 요가 없으며, 이상신호가 발생한 후 이상원인의 진단에 필요한 정보를 제공한다는 장점이 있다. 이 논문 에서는 제품의 평균수명이 감소하는 것을 탐지하는 것에 초점을 두었기 때문에, 와이블 분포의 척도모수 $\eta$ 가 감소하는 경우에 대해 모의실험을 실시하였고 그 성능을 Dickinson 등 (2014)의 CUSUM 관리도 및 Choi와 Lee (2016)의 BCUSUM 관리도와 비교하였다.

모의실험 결과 Weibull GLR 관리도의 효율은 형상모수 $\beta$, 중도절단율 $\mathrm{pc}$, 그리고 표본크기 $n$ 에 영향 
Table 4.5. Comparison of the $\mathrm{ARL}_{1}$ values for $\beta=3, \eta_{0}=1, \mathrm{pc}=0.15$ with $\mathrm{ARL}_{0} \approx 370$

\begin{tabular}{|c|c|c|c|c|c|c|}
\hline$n$ & $\eta_{1}$ & $\begin{array}{c}\text { CUSUM } \\
h_{C}=-4.55\end{array}$ & $\begin{array}{c}\text { BCUSUM } \\
h_{S}=2.159\end{array}$ & $\begin{array}{c}\text { GLR } \\
h_{\mathrm{GLR}}=5.67\end{array}$ & $\hat{\tau}$ & $\hat{\eta}_{1}$ \\
\hline \multirow{12}{*}{3} & 0.95 & 89.34 & 92.25 & 85.31 & 94.4 & 0.74 \\
\hline & 0.90 & 29.27 & 34.29 & 28.95 & 56.8 & 0.77 \\
\hline & 0.85 & 12.76 & 18.31 & 14.37 & 50.9 & 0.75 \\
\hline & 0.80 & 7.38 & 12.55 & 8.82 & 49.6 & 0.72 \\
\hline & 0.75 & 5.36 & 10.32 & 6.05 & 49.5 & 0.69 \\
\hline & 0.70 & 4.36 & 9.40 & 4.43 & 49.5 & 0.64 \\
\hline & 0.65 & 3.62 & 9.03 & 3.40 & 49.5 & 0.60 \\
\hline & 0.60 & 3.25 & 8.98 & 2.77 & 49.6 & 0.56 \\
\hline & 0.55 & 2.93 & 8.98 & 2.27 & 49.6 & 0.52 \\
\hline & 0.50 & 2.76 & 8.98 & 1.94 & 49.7 & 0.47 \\
\hline & 0.45 & 2.71 & 8.98 & 1.67 & 49.8 & 0.42 \\
\hline & 0.40 & 2.60 & 8.98 & 1.44 & 49.8 & 0.38 \\
\hline$n$ & $\eta_{1}$ & $\begin{array}{c}\text { CUSUM } \\
h_{C}=-4.68\end{array}$ & $\begin{array}{c}\text { BCUSUM } \\
h_{S}=2.244\end{array}$ & $\begin{array}{c}\text { GLR } \\
h_{\mathrm{GLR}}=5.48\end{array}$ & $\hat{\tau}$ & $\hat{\eta}_{1}$ \\
\hline \multirow{12}{*}{5} & 0.95 & 77.15 & 78.89 & 56.98 & 75.7 & 0.81 \\
\hline & 0.90 & 21.37 & 25.71 & 18.55 & 52.6 & 0.81 \\
\hline & 0.85 & 8.92 & 12.86 & 9.24 & 50.0 & 0.78 \\
\hline & 0.80 & 5.19 & 8.70 & 5.66 & 49.5 & 0.74 \\
\hline & 0.75 & 3.70 & 7.03 & 3.91 & 49.5 & 0.70 \\
\hline & 0.70 & 2.96 & 6.35 & 2.90 & 49.6 & 0.66 \\
\hline & 0.65 & 2.52 & 6.10 & 2.28 & 49.6 & 0.62 \\
\hline & 0.60 & 2.21 & 6.02 & 1.87 & 49.7 & 0.57 \\
\hline & 0.55 & 2.00 & 6.01 & 1.57 & 49.8 & 0.53 \\
\hline & 0.50 & 1.92 & 6.01 & 1.31 & 49.8 & 0.48 \\
\hline & 0.45 & 1.89 & 6.01 & 1.10 & 49.9 & 0.44 \\
\hline & 0.40 & 1.88 & 6.01 & 1.01 & 49.9 & 0.39 \\
\hline$n$ & $\eta_{1}$ & $\begin{array}{c}\text { CUSUM } \\
h_{C}=-4.71\end{array}$ & $\begin{array}{l}\text { BCUSUM } \\
h_{S}=2.25\end{array}$ & $\begin{array}{c}\text { GLR } \\
h_{\mathrm{GLR}}=5.29\end{array}$ & $\hat{\tau}$ & $\hat{\eta}_{1}$ \\
\hline \multirow{12}{*}{10} & 0.95 & 59.65 & 60.99 & 31.94 & 59.7 & 0.86 \\
\hline & 0.90 & 14.18 & 16.20 & 10.19 & 50.2 & 0.84 \\
\hline & 0.85 & 5.42 & 7.68 & 5.17 & 49.5 & 0.80 \\
\hline & 0.80 & 3.08 & 5.11 & 3.19 & 49.5 & 0.76 \\
\hline & 0.75 & 2.21 & 4.14 & 2.27 & 49.6 & 0.72 \\
\hline & 0.70 & 1.82 & 3.77 & 1.72 & 49.7 & 0.68 \\
\hline & 0.65 & 1.56 & 3.65 & 1.38 & 49.8 & 0.63 \\
\hline & 0.60 & 1.30 & 3.62 & 1.13 & 49.8 & 0.59 \\
\hline & 0.55 & 1.09 & 3.61 & 1.01 & 49.9 & 0.54 \\
\hline & 0.50 & 1.01 & 3.61 & 1.00 & 49.9 & 0.49 \\
\hline & 0.45 & 1.00 & 3.61 & 1.00 & 49.9 & 0.44 \\
\hline & 0.40 & 1.00 & 3.61 & 1.00 & 49.9 & 0.39 \\
\hline
\end{tabular}

The CUSUM and BCUSUM charts are designed to detect $\eta_{1}=0.80$.

$\mathrm{ARL}=$ average run length; CUSUM = cumulative sum; BCUSUM = binomial CUSUM; GLR = generalized likelihood ratio. 
Table 4.6. Comparison of the $\mathrm{ARL}_{1}$ values for $\beta=3, \eta_{0}=1, \mathrm{pc}=0.5$ with $\mathrm{ARL}_{0} \approx 370$

\begin{tabular}{|c|c|c|c|c|c|c|}
\hline$n$ & $\eta_{1}$ & $\begin{array}{c}\text { CUSUM } \\
h_{C}=-4.29\end{array}$ & $\begin{array}{c}\text { BCUSUM } \\
h_{S}=3.8501\end{array}$ & $\begin{array}{c}\text { GLR } \\
h_{\mathrm{GLR}}=5.51\end{array}$ & $\hat{\tau}$ & $\hat{\eta}_{1}$ \\
\hline \multirow{12}{*}{3} & 0.95 & 101.79 & 101.66 & 109.48 & 124.4 & 0.69 \\
\hline & 0.90 & 34.71 & 35.69 & 38.70 & 64.1 & 0.73 \\
\hline & 0.85 & 15.86 & 16.44 & 18.72 & 53.3 & 0.73 \\
\hline & 0.80 & 9.13 & 9.67 & 10.88 & 50.6 & 0.70 \\
\hline & 0.75 & 6.20 & 6.71 & 6.95 & 49.7 & 0.67 \\
\hline & 0.70 & 4.60 & 5.28 & 4.84 & 49.6 & 0.63 \\
\hline & 0.65 & 3.73 & 4.44 & 3.61 & 49.5 & 0.59 \\
\hline & 0.60 & 3.18 & 3.93 & 2.80 & 49.6 & 0.55 \\
\hline & 0.55 & 2.85 & 3.66 & 2.27 & 49.6 & 0.51 \\
\hline & 0.50 & 2.69 & 3.54 & 1.90 & 49.7 & 0.47 \\
\hline & 0.45 & 2.62 & 3.51 & 1.64 & 49.8 & 0.42 \\
\hline & 0.40 & 2.56 & 3.50 & 1.43 & 49.8 & 0.38 \\
\hline$n$ & $\eta_{1}$ & $\begin{array}{c}\text { CUSUM } \\
h_{C}=-4.469\end{array}$ & $\begin{array}{c}\text { BCUSUM } \\
h_{S}=4.084\end{array}$ & $\begin{array}{c}\text { GLR } \\
h_{\mathrm{GLR}}=5.32\end{array}$ & $\hat{\tau}$ & $\hat{\eta}_{1}$ \\
\hline \multirow{12}{*}{5} & 0.95 & 87.02 & 85.56 & 76.89 & 94.9 & 0.77 \\
\hline & 0.90 & 26.38 & 26.81 & 25.45 & 56.9 & 0.78 \\
\hline & 0.85 & 11.22 & 11.60 & 12.05 & 51.2 & 0.76 \\
\hline & 0.80 & 6.33 & 6.67 & 6.95 & 49.9 & 0.73 \\
\hline & 0.75 & 4.27 & 4.62 & 4.57 & 49.5 & 0.69 \\
\hline & 0.70 & 3.21 & 3.60 & 3.17 & 49.6 & 0.65 \\
\hline & 0.65 & 2.60 & 3.06 & 2.41 & 49.6 & 0.61 \\
\hline & 0.60 & 2.22 & 2.77 & 1.89 & 49.7 & 0.57 \\
\hline & 0.55 & 1.99 & 2.65 & 1.55 & 49.8 & 0.52 \\
\hline & 0.50 & 1.90 & 2.60 & 1.29 & 49.8 & 0.48 \\
\hline & 0.45 & 1.87 & 2.60 & 1.08 & 49.9 & 0.43 \\
\hline & 0.40 & 1.86 & 2.60 & 1.00 & 49.9 & 0.39 \\
\hline$n$ & $\eta_{1}$ & $\begin{array}{c}\text { CUSUM } \\
h_{C}=-4.60\end{array}$ & $\begin{array}{c}\text { BCUSUM } \\
h_{S}=4.214\end{array}$ & $\begin{array}{c}\text { GLR } \\
h_{\mathrm{GLR}}=5.12\end{array}$ & $\hat{\tau}$ & $\hat{\eta}_{1}$ \\
\hline \multirow{12}{*}{10} & 0.95 & 69.78 & 65.90 & 45.17 & 69.8 & 0.84 \\
\hline & 0.90 & 17.45 & 17.73 & 13.91 & 51.8 & 0.83 \\
\hline & 0.85 & 6.73 & 7.04 & 6.69 & 49.5 & 0.79 \\
\hline & 0.80 & 3.76 & 3.95 & 3.95 & 49.5 & 0.76 \\
\hline & 0.75 & 2.57 & 2.70 & 2.61 & 49.6 & 0.72 \\
\hline & 0.70 & 1.95 & 2.16 & 1.89 & 49.7 & 0.67 \\
\hline & 0.65 & 1.61 & 1.90 & 1.45 & 49.7 & 0.63 \\
\hline & 0.60 & 1.34 & 1.80 & 1.17 & 49.8 & 0.59 \\
\hline & 0.55 & 1.11 & 1.78 & 1.03 & 49.9 & 0.54 \\
\hline & 0.50 & 1.01 & 1.76 & 1.00 & 49.9 & 0.49 \\
\hline & 0.45 & 1.00 & 1.75 & 1.00 & 49.9 & 0.44 \\
\hline & 0.40 & 1.00 & 1.75 & 1.00 & 49.9 & 0.39 \\
\hline
\end{tabular}

The CUSUM and BCUSUM charts are designed to detect $\eta_{1}=0.80$.

$\mathrm{ARL}=$ average run length; CUSUM $=$ cumulative sum; BCUSUM $=$ binomial CUSUM; GLR = generalized likelihood ratio. 
Table 4.7. Comparison of the $\mathrm{ARL}_{1}$ values for $\beta=3, \eta_{0}=1, \mathrm{pc}=0.95$ with $\mathrm{ARL}_{0} \approx 370$

\begin{tabular}{|c|c|c|c|c|c|c|}
\hline$n$ & $\eta_{1}$ & $\begin{array}{c}\text { CUSUM } \\
h_{C}=-2.81\end{array}$ & $\begin{array}{l}\text { BCUSUM } \\
h_{S}=3.82\end{array}$ & $\begin{array}{c}\text { GLR } \\
h_{\mathrm{GLR}}=4.76\end{array}$ & $\hat{\tau}$ & $\hat{\eta}_{1}$ \\
\hline \multirow{12}{*}{3} & 0.95 & 182.36 & 177.07 & 222.59 & 264.6 & 0.47 \\
\hline & 0.90 & 97.55 & 96.02 & 129.98 & 191.6 & 0.50 \\
\hline & 0.85 & 56.00 & 54.94 & 76.97 & 104.8 & 0.53 \\
\hline & 0.80 & 34.80 & 33.98 & 47.09 & 79.1 & 0.54 \\
\hline & 0.75 & 22.84 & 22.25 & 29.05 & 64.0 & 0.54 \\
\hline & 0.70 & 15.68 & 15.31 & 18.75 & 57.3 & 0.52 \\
\hline & 0.65 & 11.14 & 11.07 & 12.67 & 53.8 & 0.51 \\
\hline & 0.60 & 8.18 & 8.06 & 8.39 & 51.5 & 0.49 \\
\hline & 0.55 & 5.98 & 5.97 & 5.81 & 50.6 & 0.46 \\
\hline & 0.50 & 4.54 & 4.53 & 4.08 & 50.0 & 0.44 \\
\hline & 0.45 & 3.49 & 3.47 & 2.94 & 49.8 & 0.41 \\
\hline & 0.40 & 2.73 & 2.71 & 2.16 & 49.7 & 0.37 \\
\hline$n$ & $\eta_{1}$ & $\begin{array}{c}\text { CUSUM } \\
h_{C}=-3.461\end{array}$ & $\begin{array}{c}\text { BCUSUM } \\
h_{S}=4.3094\end{array}$ & $\begin{array}{c}\text { GLR } \\
h_{\mathrm{GLR}}=4.62\end{array}$ & $\hat{\tau}$ & $\hat{\eta}_{1}$ \\
\hline \multirow{12}{*}{5} & 0.95 & 169.31 & 155.20 & 196.02 & 231.2 & 0.54 \\
\hline & 0.90 & 80.82 & 76.40 & 100.73 & 128.4 & 0.58 \\
\hline & 0.85 & 43.53 & 41.49 & 55.01 & 84.6 & 0.59 \\
\hline & 0.80 & 26.15 & 24.77 & 31.24 & 64.8 & 0.59 \\
\hline & 0.75 & 16.82 & 16.00 & 19.39 & 57.0 & 0.58 \\
\hline & 0.70 & 11.52 & 11.00 & 12.34 & 53.3 & 0.56 \\
\hline & 0.65 & 8.25 & 7.88 & 8.50 & 51.5 & 0.54 \\
\hline & 0.60 & 6.08 & 5.84 & 5.67 & 50.4 & 0.52 \\
\hline & 0.55 & 4.55 & 4.38 & 3.93 & 49.8 & 0.49 \\
\hline & 0.50 & 3.46 & 3.36 & 2.81 & 49.7 & 0.46 \\
\hline & 0.45 & 2.67 & 2.62 & 2.02 & 49.7 & 0.42 \\
\hline & 0.40 & 2.09 & 2.08 & 1.48 & 49.8 & 0.38 \\
\hline$n$ & $\eta_{1}$ & $\begin{array}{c}\text { CUSUM } \\
h_{C}=-3.60\end{array}$ & $\begin{array}{c}\text { BCUSUM } \\
h_{S}=4.940\end{array}$ & $\begin{array}{c}\text { GLR } \\
h_{\mathrm{GLR}}=4.55\end{array}$ & $\hat{\tau}$ & $\hat{\eta}_{1}$ \\
\hline \multirow{12}{*}{10} & 0.95 & 133.85 & 132.05 & 154.18 & 182.7 & 0.63 \\
\hline & 0.90 & 56.73 & 56.59 & 66.28 & 92.5 & 0.67 \\
\hline & 0.85 & 28.11 & 27.89 & 33.41 & 65.1 & 0.67 \\
\hline & 0.80 & 15.99 & 15.85 & 18.68 & 55.5 & 0.66 \\
\hline & 0.75 & 10.14 & 10.10 & 11.47 & 52.0 & 0.64 \\
\hline & 0.70 & 6.92 & 6.91 & 7.42 & 50.5 & 0.61 \\
\hline & 0.65 & 5.01 & 4.99 & 4.97 & 49.9 & 0.59 \\
\hline & 0.60 & 3.71 & 3.74 & 3.45 & 49.8 & 0.55 \\
\hline & 0.55 & 2.83 & 2.84 & 2.44 & 49.7 & 0.52 \\
\hline & 0.50 & 2.19 & 2.20 & 1.77 & 49.7 & 0.48 \\
\hline & 0.45 & 1.72 & 1.74 & 1.33 & 49.8 & 0.44 \\
\hline & 0.40 & 1.36 & 1.37 & 1.09 & 49.8 & 0.39 \\
\hline
\end{tabular}

The CUSUM and BCUSUM charts are designed to detect $\eta_{1}=0.80$.

$\mathrm{ARL}=$ average run length; CUSUM = cumulative sum; BCUSUM = binomial CUSUM; GLR = generalized likelihood ratio. 
을 받으며, $\mathrm{pc}$ 가 높지 않을 경우에는 $\beta$ 와 $n$ 이 커질수록 Weibull GLR 관리도의 성능이 향상되는 것을 알 수 있었다.

결론적으로 $\beta$ 가 작고 $\mathrm{pc}$ 가 높지 않은 경우에는 Dickinson 등 (2014)의 CUSUM 관리도, $\mathrm{pc}$ 가 아주 높 은 경우에는 Choi와 Lee (2016)의 BCUSUM 관리도, 그리고 $\beta$ 가 크고 $\mathrm{pc}$ 가 아주 높지 않은 경우에는 이 논문에서 제안된 Weibull GLR 관리도의 사용을 추천하고자 한다.

\section{References}

Choi, M. J. and Lee, J. (2016). A binomial CUSUM chart for monitoring type I right-censored Weibull lifetimes, The Korean Journal of Applied Statistics, 29, 823-833.

Dickinson, R. M., Olteanu Roberts, D. A., Driscoll, A. R., Woodall, W. H., and Vining, G. G. (2014). CUSUM charts for monitoring the characteristic life of censored Weibull lifetimes, Journal of Quality Technology, 46, 340-358.

Kao, J. H. K. (1959). A graphical estimation of mixed Weibull parameters in life-testing of electron tubers, Technometrics, 1, 389-407.

Lawless, J. F. (2003). Statistical Models and Methods for Lifetime Data (2nd ed.), John Wiley \& Sons, Inc., Hoboken, NJ.

Lieblein, J. and Zelen, M. (1956). Statistical investigation of the fatigue life of deep-groove ball bearings, Journal of Research of the National Bureau of Standards, 57, 273-316.

Olteanu, D. A. (2010). Cumulative Sum Control Charts for Censored Reliability Data, Ph.D. Dissertation, Virginia Tech.

Reynolds, Jr., M. R. and Lou, J. (2010). An evaluation of a GLR control chart for monitoring the process mean, Journal of Quality Technology, 42, 287-310.

Reynolds, Jr., M. R., Lou, J., Lee, J., and Wang, S. (2013). The design of GLR control charts for monitoring the process mean and variance, Journal of Quality Technology, 45, 34-60.

Ryan, A. G., Wells, L. J., and Woodall, W. H. (2011). Methods for monitoring multiple proportions when inspecting continuously, Journal of Quality Technology, 43, 237-248.

Steiner, S. H. and MacKay, R. J. (2001). Monitoring processes with data censored owing to competing risks by using exponentially weighted moving average control charts, Journal of the Royal Statistical Society, Series C: Applied Statistics, 50, 293-302.

Zhang, L. and Chen, G. (2004). EWMA charts for monitoring the mean of censored Weibull lifetimes, Journal of Quality Technology, 36, 321-328. 


\title{
제1형 우측중도절단된 와이블 수명자료를 모니터링하는 GLR 관리도
}

\author{
한성원 $^{a} \cdot$ 이재헌 ${ }^{a, 1}$ \\ ${ }^{a}$ 중앙대학교 응용통계학과
}

(2017년 07월 17일 접수, 2017년 08월 23일 수정, 2017년 08월 23일 채택)

\section{요 약}

와이블 분포는 제품의 물리적 특성을 잘 반영하고 고장률이 증가하거나 감소하는 경우 모두를 나타낼 수 있기 때문에 재품의 수명을 모델링할 때 가장 많이 사용되는 분포이다. 일반적으로 수명시험에서 표본의 모든 제품의 수명이 측 정될 때까지 시험을 진행하는 것이 원칙이지만 여러 가지 시간적 또는 비용적인 제약으로 인해 시험을 중도절단 하는 경우가 빈번하게 발생한다. 이 논문에서는 제품의 수명 데이터가 와이블 분포를 따르고 제 1 형 우측중도절단된 경우 척도모수의 변화를 탐지하는 Weibull generalized likelihood ratio (GLR) 관리도 절차를 제안하였다. 모의실험을 실시하여 제안한 GLR 관리도와 기존에 제안된 두 가지 CUSUM 관리도의 성능을 average run length (ARL)을 이용하여 비교하였다. 그 결과 이 논문에서 제안한 GLR 관리도는 형상모수와 표본의 크기가 크고 중도절단율이 아 주 높지 않은 경우 척도모수의 다양한 변화를 탐지하는데 효율적임을 알 수 있었다.

주요용어: 와이블 분포, 일반화 우도비(GLR) 관리도, 제1형 우측중도절단 데이터, 평균런길이

이 논문은 2016년도 중앙대학교 CAU GRS 지원에 의하여 작성되었고, 2017년도 정부(교육부)의 재원으로 한국연구재단의 지원을 받아 수행된 기초연구사업임 (No. 2017R1D1A1B03029035).

${ }^{1}$ 교신저자: (06974) 서울특별시 동작구 흑석로 84, 중앙대학교 응용통계학과. E-mail: jaeheon@cau.ac.kr 